\title{
Concentración de adiponectina posterior a reducción de masa corporal en un grupo de mujeres obesas premenopáusicas en Lima, Perú
}

Wilser Andrés García-Quispes 1; Dina Torres Gonzales 2,5; Carlos Patricio Padilla Rojas ${ }^{3}$; Frank Lizaraso-Soto ${ }^{2}$; Jorge Calderón Ticona ${ }^{4}$; José Solis Villanueva ${ }^{4}$; Mónica Yolanda Paredes Anaya*1

\section{RESUMEN}

Objetivo: Investigar el cambio en la concentración de adiponectina circulante en plasma en 23 mujeres obesas premenopáusicas de la ciudad de Lima luego de la reducción de la masa corporal como resultado de un programa de actividad física aeróbica y una dieta baja en calorías.

Materiales y métodos: Se llevó a cabo un estudio analítico y cuasi-experimental con el grupo de mujeres obesas. Adicionalmente, 24 mujeres se consideraron como control o de referencia de la concentración de adiponectina y otros marcadores bioquímicos. La cuantificación de adiponectina se realizó mediante la prueba de ELISA. La concentración de glucosa en ayunas en sangre, colesterol, lipoproteína de alta densidad y triglicéridos fueron cuantificados mediante análisis clínicos de rutina.

Resultados: Antes del programa el grupo control mostró altos valores de adiponectina (mediana: 8,54 $\mu \mathrm{g} / \mathrm{mL}$; rango: $6,14 \mu \mathrm{g} / \mathrm{mL}$ a $13,49 \mu \mathrm{g} / \mathrm{mL}$ ) en comparación con el grupo obesidad (mediana: 7,03 $\mu \mathrm{g} / \mathrm{mL}$, rango: 3;77 $\mu \mathrm{g} / \mathrm{mL}$ a $17,23 \mu \mathrm{g} /$ $\mathrm{mL})$; sin embargo, la diferencia no fue estadísticamente significativa $(P=0.0563)$. Luego de la finalización del programa se observó que el grupo obesidad presentó una reducción estadísticamente significativa del índice de masa corporal $(\mathrm{P}=$ 5.98e-08) y de la circunferencia abdominal $(P=1.55 e-08)$ así como un incremento estadísticamente significativo de los niveles de adiponectina (mediana, $8.79 \mu \mathrm{g} / \mathrm{mL}$; rango, $5.50 \mu \mathrm{g} / \mathrm{mL}$ a $19.37 \mu \mathrm{g} / \mathrm{mL})(P=0.0127)$.

Conclusiones: Basándonos en los resultados, concluimos que en mujeres obesas premenopáusicas la concentración de adiponectina se incrementa cuando la masa corporal se reduce como resultado de actividad física aeróbica y una dieta baja en calorías.

Palabras clave: Adiponectina; Actividad física; Índice de masa corporal; Programas de reducción de peso; Dieta reductora (Fuente: DeCS BIREME).

\section{Adiponectin concentration after a body mass reduction in a group of premenopausal obese women living in Lima, Peru}

\section{ABSTRACT}

Objective: To investigate the changes in the plasma-circulating adiponectin concentration in 23 premenopausal obese women living in Lima after a body mass reduction as a result of an aerobic physical activity program and a low-calorie diet. Materials and methods: An analytical and quasi-experimental study was conducted in a group of obese women. In addition, another 24 women were considered as control or reference group for comparing their adiponectin concentration and other biochemical markers. The quantification of adiponectin was carried out using the ELISA test. Fasting blood glucose concentration, cholesterol, high-density lipoprotein and triglycerides levels were quantified by routine clinical analysis. Results: Before beginning the program, the control group showed high adiponectin levels (median, $8.54 \mu \mathrm{g} / \mathrm{mL} ;$ range, $6.14 \mu \mathrm{g} /$ $\mathrm{mL}$ to $13.49 \mu \mathrm{g} / \mathrm{mL}$ ) compared to the obesity group (median, $7.03 \mu \mathrm{g} / \mathrm{mL} ;$ range, $3.77 \mu \mathrm{g} / \mathrm{mL}$ to $17.23 \mu \mathrm{g} / \mathrm{mL}$ ). However, the difference was not statistically significant $(P=0.0563)$. Once the program was finished, the obesity group showed a statistically significant reduction in the body mass index $(P=5.98 \mathrm{e}-08)$ and abdominal circumference $(P=1.55 \mathrm{e}-08)$, and a statistically significant increase in the adiponectin levels (median, $8.79 \mu \mathrm{g} / \mathrm{mL}$; range, $5.50 \mu \mathrm{g} / \mathrm{mL}$ to $19.37 \mu \mathrm{g} / \mathrm{mL})(P=0.0127)$.

Conclusions: Based on the results, we conclude that the adiponectin concentration in premenopausal obese women increases when the body mass is reduced as a result of aerobic physical activity and a low-calorie diet.

Keywords: Adiponectin; Exercise; Body mass index; Weight reduction programs; Diet, reducing (Source: MeSH NLM).

1. Universidad Nacional Mayor de San Marcos. Facultad de Ciencias Biológicas, Laboratorio de Genética de Enfermedades Metabólicas. Lima, Perú.

2. Universidad de San Martín de Porres, Facultad de Medicina Humana. Lima, Perú.

3. Instituto Nacional de Salud Perú, Laboratorio de Biotecnología y Biología Molecular. Lima,Perú.

4. Hospital Nacional Arzobispo Loayza. Servicio de Endocrinología. Lima, Perú

5. Instituto Nacional Materno Perinatal, Departamento de Patología, Laboratorio de Citogenética. Lima, Perú.

*Autor corresponsal. 


\section{INTRODUCCIÓN}

De acuerdo con la Organización Mundial de la Salud (OMS), la obesidad ha triplicado su prevalencia desde el año 1975 , aunque es un cuadro prevenible ${ }^{(1)}$. La obesidad es el resultado una interacción compleja entre factores endocrinos, psicológicos, ambientales y genéticos ${ }^{(2,3)}$ que se relacionan a una serie de cambios metabólicos con múltiples efectos que llevan al desarrollo de otras patologías como diabetes tipo 2, enfermedad cardiovascular o cáncer (4). Esto, además del impacto emocional en los pacientes, supone un efecto directo en el gasto de salud pública ${ }^{(5)}$ así como efectos indirectos en el ambiente ${ }^{(6)}$.

Existen marcadores bioquímicos asociados con cambios metabólicos que ocurren durante el desarrollo de la obesidad, entre estos se encuentra la adiponectina, una adipoquina secretada por el tejido adiposo. Esta proteína constituye entre $0,01 \%$ y 0,05 \% de todas las proteínas del plasma ${ }^{(7,8)}$. En humanos el gen que codifica esta proteína es adiponectin, C1Q and collagen domain containing (ADIPOQ) localizado en el brazo largo del cromosoma 3 (3q27.3). Desde el año 2000, diferentes estudios evidencian que los niveles altos de adiponectina se asocian con un buen estado de salud; sin embargo, este hecho no es constante entre las distintas investigaciones ${ }^{(9-12)}$. Otros estudios, en animales y humanos, han demostrado la relación entre la concentración de adiponectina y una serie de cambios metabólicos y hormonales que incluyen obesidad, síndrome metabólico, diabetes tipo 2, enfermedad cardiovascular, cáncer, e incluso, infecciones virales (9,13-18). A partir de esta evidencia, se ha sugerido que la desregulación de los niveles de adiponectina podría ser la causa del desarrollo de enfermedad cardiovascular o procesos inflamatorios ${ }^{(19,20)}$. Así, la adiponectina se considera un biomarcador del estado de salud. De hecho, algunas investigaciones tienen como objetivo el estudio de la administración exógena de adiponectina y su efecto en el mejoramiento de la condición del paciente ${ }^{(9,21,22)}$, no solo en enfermedades metabólicas, sino también en distrofia muscular de Duchenne ${ }^{(23)}$ o mal de Alzheimer (24). Revisiones y metaanálisis recientes encuentran algunas discrepancias entre estudios con respecto al uso de adiponectina como un biomarcador de enfermedades metabólicas por lo que todavía se recomienda incrementar el número de investigaciones de modo que este aspecto pueda ser aclarado $(7,13,19,25,26)$.

El objetivo de este estudio fue investigar el cambio en la concentración de adiponectina circulante en plasma en 23 mujeres premenopáusicas de la ciudad de Lima luego de la reducción de la masa corporal como resultado de un programa de reducción de peso, que incluyó una rutina de actividad física aeróbica y una dieta baja en calorías.

\section{MATERIALES Y MÉTODOS}

Diseño y población de estudio

Se llevó a cabo un estudio analítico y cuasi-experimental (antes-después) para determinar el efecto de la reducción de masa corporal sobre la concentración de adiponectina en 23 mujeres premenopáusicas con diagnóstico de obesidad dado por el Servicio de Endocrinología del Hospital Nacional Arzobispo Loayza (20 mujeres con $25 \mathrm{~kg} /$ $\mathrm{m} 2<\mathrm{IMC} \leq 40 \mathrm{~kg} / \mathrm{m}^{2}$ y 3 mujeres con $\mathrm{IMC}>40 \mathrm{~kg} / \mathrm{m2}$ ). Las mujeres fueron informadas sobre la investigación y se les solicitó su participación. Adicionalmente, 24 mujeres no obesas (IMC $\leq 25 \mathrm{~kg} / \mathrm{m}^{2}$ ) fueron consideradas como controles, únicamente para tener referencia de los valores normales de adiponectina y otros marcadores bioquímicos. Todas las participantes ingresaron al estudio luego de la firma del consentimiento informado.

\section{Variables y mediciones}

Por cada participante se tomaron dos muestras de sangre $(5 \mathrm{~mL})$ en tubos vacuotainer con EDTA como anticoagulante. En menos de una hora, una de las muestras fue transportada a $4{ }^{\circ} \mathrm{C}$ al laboratorio para su análisis. El plasma fue separado por centrifugación y almacenado a $-80^{\circ} \mathrm{C}$ hasta el momento de la cuantificación de adiponectina. La segunda muestra se usó para determinar la concentración de glucosa en ayunas, colesterol, lipoproteína de alta densidad, triglicéridos y microalbuminuria. Esta cuantificación se realizó mediante análisis clínicos de rutina realizados en el Servicio de Endocrinología del Hospital Nacional Arzobispo Loayza.

La concentración de adiponectina en plasma determinó mediante ELISA con el kit Adiponectin Human ELISA (Número de catálogo: KHP0041), de acuerdo con las recomendaciones del fabricante. Por cada muestra se realizaron tres réplicas. La concentración de adiponectina se estimó mediante el reemplazo del valor de absorbancia en la ecuación de regresión y la multiplicación por el factor de dilución. La media de las tres lecturas de absorbancia se usó como valor final.

Programa de reducción de masa corporal

La reducción de la masa corporal se llevó a cabo mediante una rutina de actividad física y una dieta baja en calorías. Este programa se aplicó para mujeres en el grupo de obesidad que participaron en el programa de actividad física aeróbica durante 10 semanas. Específicamente, las mujeres realizaron una sesión aeróbica de una hora, tres veces por semana, durante un período de 10 semanas. Cada sesión involucró calentamiento muscular (10 minutos), estiramiento muscular (10 minutos), ejercicios aeróbicos basados en caminatas rápidas (15 minutos con un incremento de 5 minutos por semana hasta alcanzar 30 minutos) y enfriamiento y ejercicios de estiramiento (10 minutos). Esta actividad fue supervisada por un terapeuta del Servicio de Terapia Física y Rehabilitación del Hospital Nacional Arzobispo Loayza. 
La dieta baja en calorías (aproximadamente $1200 \mathrm{kcal} /$ día) fue diseñada por un nutricionista. Todas las participantes prepararon su comida (ayuno, desayuno, almuerzo, cena) de acuerdo con las indicaciones del nutricionista.

\section{Análisis estadístico}

Todas las variables numéricas fueron descritas con la media, mediana, desviación estándar, mínimo y máximo (Tabla 1). La prueba de Shapiro-Wilk se usó para evaluar la normalidad de la distribución de las variables. Los resultados de esta prueba se usaron como criterio para la elección de las pruebas estadísticas para la comparación entre los grupos control y obesidad (muestras independientes), así como para la comparación en el grupo obesidad antes y después del programa de actividad física y dieta (muestras apareadas). Los resultados de las pruebas estadísticas se describen con las diferencias entre los grupos y su respectivo intervalo de confianza del $95 \%$ (indicado entre corchetes [ ]) así como con el valor de probabilidad (P).

Para estudiar la correlación entre la concentración de adiponectina y todas las variables numéricas se elaboraron gráficos de dispersión acompañados de líneas suavizadas de tendencia (lowess, locally weighted regression and smoothing scatter-plots). El coeficiente de correlación de Pearson ( $r$ ) solo fue usado cuando las variables involucradas en la correlación seguían una distribución normal, de lo contrario se usó el coeficiente de Spearman (rho). Los intervalos de confianza de los coeficientes solo fueron calculados para los coeficientes de Pearson. Todos los gráficos y análisis estadísticos se elaboraron con el lenguaje de programación $R$ versión $3.4 .0^{(27)}$ bajo el entorno gráfico RStudio versión 1.0.143 ${ }^{(28)}$.

\section{Consideraciones éticas}

La investigación fue aprobada por el Comité de Ética para la Investigación de la Universidad de San Martín de Porres (Oficio $\mathrm{N}^{\circ} 1128$-CEI-FMH-USMP con fecha 06 de mayo de 2010). Las participantes ingresaron al estudio luego de la firma del consentimiento informado.

\section{RESULTADOS}

La descripción general de las variables evaluadas en ambos grupos (control y obesidad, antes del inicio del programa de reducción de masa corporal) se describen en la Tabla 1.

Tabla 1. Características generales de los grupos control y obesidad iniciales

\begin{tabular}{lcccc} 
& \multicolumn{2}{c}{ Control } & \multicolumn{2}{c}{ Obesidad } \\
& Media (SD) & Mediana (min,max) & Media (SD) & Mediana (min,max) \\
Edad (años) & $42,8(5,3)$ & $42,5(35,50)$ & $43,1(6,5)$ & $43(35,56)$ \\
Peso $(\mathrm{kg})$ & $57,0(5,6)$ & $55,0(48,66)$ & $85,2(15,0)$ & $87,9(56,117)$ \\
\hline Altura $(\mathrm{m})$ & $1,58(0.05)$ & $1,58(1,48,1,69)$ & $1,57(0,08)$ & $1,55(1,40,1,71)$ \\
IMC $(\mathrm{kg} / \mathrm{m} 2)$ & $22,8(1.4)$ & $22,8(20,4,25,0)$ & $34,7(5.5)$ & $34,1(25,6,48.9)$ \\
CA $(\mathrm{cm})$ & $80,9(4.0)$ & $81,5(70,88)$ & $106,5(10.6)$ & $109,0(90,125)$ \\
Glucosa $(\mathrm{mg} / \mathrm{dl})$ & $84,8(12.6)$ & $84,5(61,110)$ & $94,7(13,2)$ & $93,0(74,130)$ \\
Colesterol $(\mathrm{mg} / \mathrm{dl})$ & $202,9(43.5)$ & $204(139,314)$ & $204,7(37,4)$ & $206(128,284)$ \\
HDL $(\mathrm{mg} / \mathrm{dl})$ & $50,8(15.6)$ & $46,5(24,93)$ & $45,3(11.3)$ & $44,0(29,66)$ \\
TG $(\mathrm{mg} / \mathrm{dl})$ & $137,3(73,6)$ & $121,5(45,324)$ & $143,1(47-3)$ & $139,0(77,274)$ \\
MA $(\mathrm{mg} / \mathrm{dl})$ & $9,7(17,6)$ & $5,2(0,39,81,8)$ & $18,0(29.3)$ & $6,1(0,28,119.2)$ \\
ADP $(\mu \mathrm{gg} / \mathrm{mL})$ & $8,7(1,89)$ & $8,5(6,15,13,49)$ & $7,8(3,27)$ & $7,03(3,77,17,23)$
\end{tabular}

IMC: Índice de masa corporal; CA: circunferencia abdominal; HDL: lipoproteína de alta densidad; TG: triglicéridos; MA: microalbuminuria; ADP: adiponectina.

a Valores antes del programa de actividad física y dieta.

SD: desviación estándar; min: mínimo; max: máximo.

Con respecto a la concentración de adiponectina, en el grupo control la mediana fue $8,54 \mu \mathrm{g} / \mathrm{mL}$ mientras que en el grupo obesidad (antes del programa) la mediana fue $7,03 \mu \mathrm{g} / \mathrm{mL}$. Sin embargo, esta diferencia no es estadísticamente significativa (Wilcoxon rank sum test, diferencia: 1.35 [-0.03 a 2.68], $\mathrm{P}=0.0563$ ). Asimismo, en contraste con el grupo control, el grupo obesidad presentó una mayor dispersión de los valores de concentración de adiponectina (Figura 1d). 
En el grupo obesidad la concentración de glucosa fue el único marcador bioquímico que mostró una diferencia estadísticamente significativa entre grupos (Welch t-test, diferencia: $9,99 \mathrm{mg} / \mathrm{dL}[2,41 \mathrm{mg} / \mathrm{dL}$ a $17,57 \mathrm{mg} / \mathrm{dL}], \mathrm{P}=$ 0.011 ) como se observa en la figura $1 \mathrm{a}$.

El grupo control mostró bajos valores de IMC y CA en comparación con el grupo obesidad (Figura 1b y 1c). Además, aunque el grupo obesidad mostró una reducción estadísticamente significativa del IMC y CA después del programa, estos valores todavía son altos en comparación
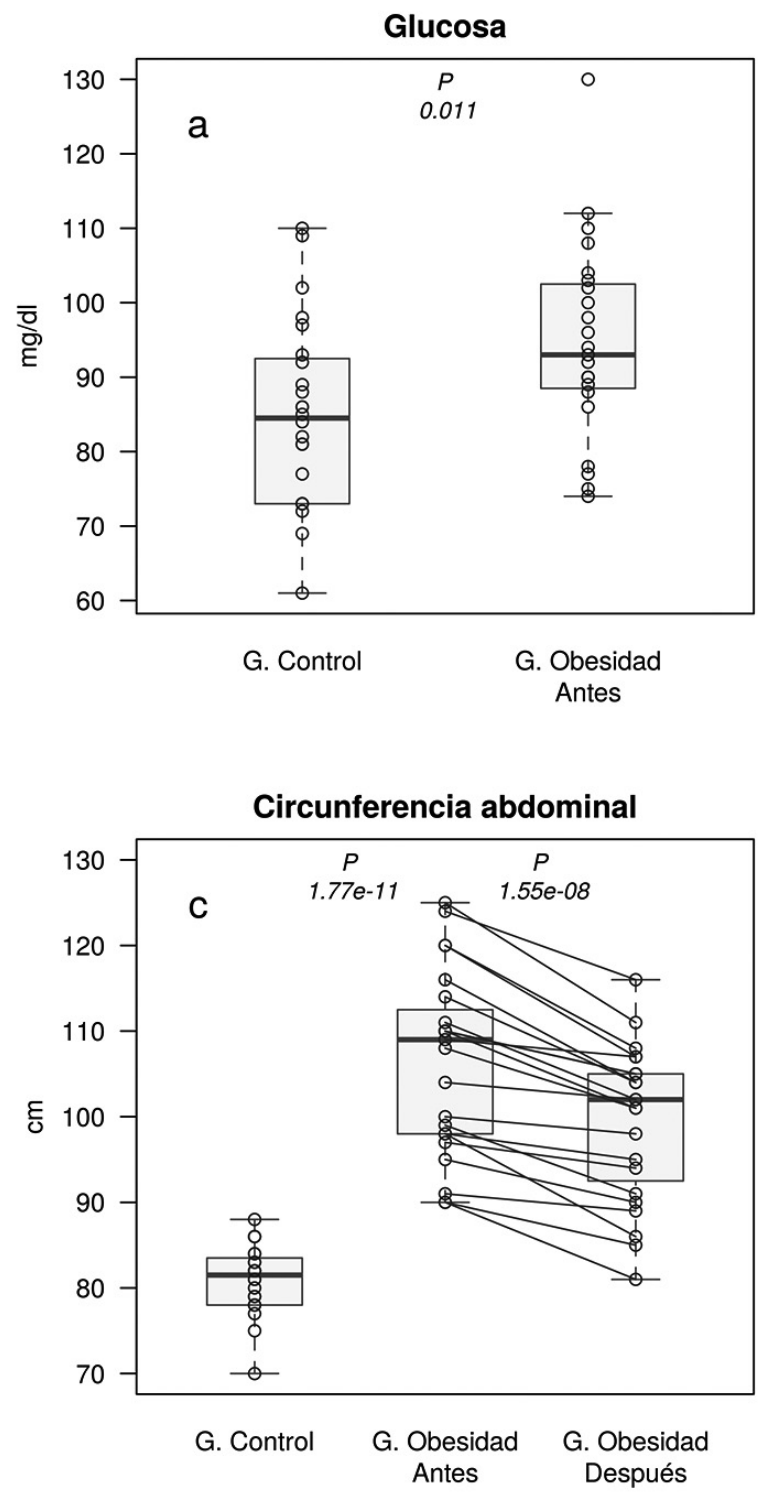

con el grupo control (Figura 1b y 1c).

La concentración de adiponectina en el grupo obesidad antes y después del programa mostró un aumento estadísticamente significativo (Wilcoxon signed rank test, diferencia: 1,82 [0,38 a 3,40], $P=0,0127$ ). Solo cinco mujeres mostraron una reducción en la concentración de adiponectina después del programa (Figura 1d). En la misma figura, se puede observar que la mediana después del programa es similar a la mediana en el grupo de control.
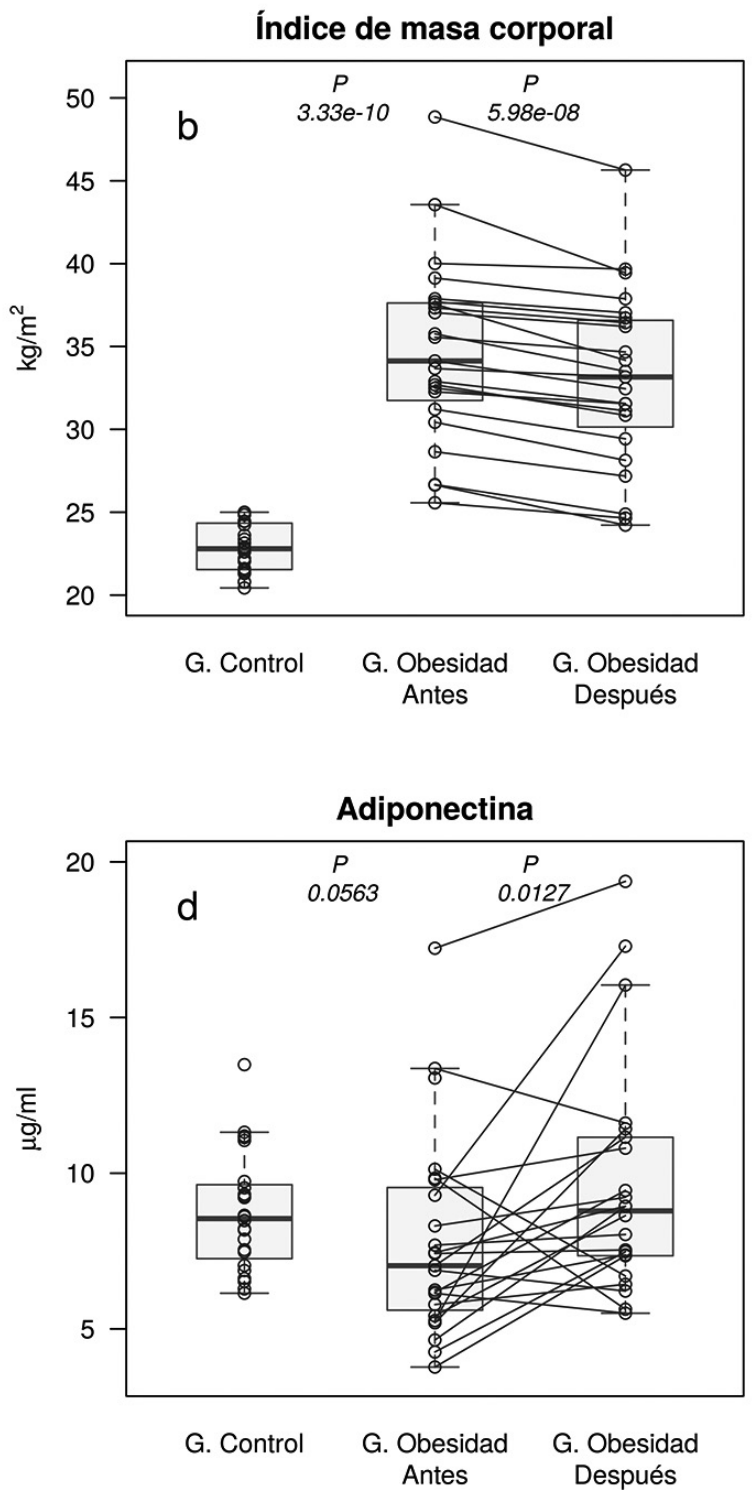

Figura 1. Comparación de concentración de glucosa, marcadores de obesidad y concentración de adiponectina entre grupos. (a) Concentración de glucosa entre grupos (Welch t-test, diferencia: $9,99 \mathrm{mg} / \mathrm{dL}[2,41 \mathrm{mg} / \mathrm{dL}$ to $17,57 \mathrm{mg} / \mathrm{dL}], P=0.011$ ). (b) Índice de masa corporal en el grupo control versus el grupo obesidad antes del programa (Welch t-test, diferencia: $11,86 \mathrm{~kg} / \mathrm{m} 2$ [9,42 kg/m² to $14,31 \mathrm{~kg} / \mathrm{m}^{2}$ ], $P=333 \mathrm{e}-10$ ). Las líneas muestran el cambio en el índice de masa corporal antes y después del programa (Paired $\mathrm{t}$-test, 
diferencia: $1,61 \mathrm{~kg} / \mathrm{m}^{2}\left[1,19 \mathrm{~kg} / \mathrm{m} 2\right.$ to $\left.2,03 \mathrm{~kg} / \mathrm{m}^{2}\right], P=5.98 \mathrm{e}-08$ ). (c) Circunferencia abdominal en los grupos control y obesidad antes del programa (Welch t-test, diferencia: $25,56 \mathrm{~cm}[20.71 \mathrm{~cm}$ to $30.41 \mathrm{~cm}], P=1.77 \mathrm{e}-11$ ). Las líneas muestran el cambio en la circunferencia abdominal antes y después del programa (Paired t-test, diferencia: 7,04 cm [5,36 cm to $8,73 \mathrm{~cm}], P=1.554 \mathrm{e}-08$ ). (d) Concentración de adiponectina entre los grupos control y obesidad antes del programa (Wilcoxon rank sum test, diferencia: 1,35 [-0,03 to 2,68], $P$ $=0.0563$ ). Las líneas muestran el cambio en los niveles de adiponectina antes y después del programa (Wilcoxon signed rank test, diferencia: 1,82 [0.38 to 3.40$], P=0.0127$ ).

Solo se encontró una correlación inversa y estadísticamente significativa entre las concentraciones de adiponectina y glucosa en el grupo control $(r=-0.42[-0.70$ a -0.02$]$, $P=0.0409)$. En el grupo obesidad no se observó ninguna correlación estadísticamente significativa que involucre la concentración de adiponectina antes o después del programa.

\section{DISCUSIÓN}

El presente estudio evalúa el cambio en la concentración de adiponectina en mujeres obesas pre-menopáusicas luego de un programa de reducción de masa corporal que involucra actividad física aeróbica y una dieta baja en calorías. Dentro de las variables bioquímicas evaluadas era esperado encontrar a la glucosa como biomarcador de obesidad debido a que esta enfermedad también se encuentra relacionada con el desarrollo de diabetes tipo $2{ }^{(29)}$. La adiponectina, que constituye el $0,01 \%$ al $0,05 \%$ de todas las proteínas circulantes en plasma ${ }^{(7,8)}$, existe como monómero, trímero de bajo peso molecular (LMW), hexámero de mediano peso molecular (MMW) y como multímero de alto peso molecular (HMW) que comprende al menos 18 monómeros $(26,30,31)$. Sin embargo, parece que la combinación de HMW y MMW es la más relevante en la respuesta inflamatoria ya que esta combinación incrementa la liberación de interleucina-6, interleucina-8 y la metaloproteinasa MMP$3^{(32)}$. Son varios los estudios que reportan asociación entre los niveles de adiponectina y diferentes enfermedades como enfermedad cardiovascular, obesidad, síndrome metabólico, diabetes tipo 2, algunos tipos de cánceres e infecciones virales, sin embargo, todavía existe un debate abierto en este tema por lo que es necesario llevar a cabo más estudios ${ }^{(7,14,17,18,33-37)}$.

El rango normal de concentración de adiponectina está entre $1,9 \mu \mathrm{g} / \mathrm{mL}$ a $17 \mu \mathrm{g} / \mathrm{mL}$ o entre $2 \mu \mathrm{g} / \mathrm{mL}$ a $20 \mu \mathrm{g} /$ $\mathrm{mL}$ en individuos no obesos ${ }^{(7,12)}$. Algunos autores reportan un rango más amplio $(3 \mu \mathrm{g} / \mathrm{ml} \text { a } 30 \mu \mathrm{g} / \mathrm{ml})^{(9,14)}$. Existen diferencias en los niveles de adiponectina entre personas obsesas y no obesas. Por ejemplo, en individuos obesos se ha observado que la concentración de adiponectina es menor en comparación con individuos no obesos $(3,7 \mu \mathrm{g} /$ $\mathrm{ml}$ en promedio) ${ }^{(12)}$. En nuestro estudio, los niveles de adiponectina encontrados en el grupo control (mediana $=$ $8,54 \mu \mathrm{g} / \mathrm{mL}$ ) concuerdan con el rango reportado por los estudios anteriormente mencionados. Además, las mujeres obesas mostraron niveles más bajos de adiponectina (mediana $=7,03 \mu \mathrm{g} / \mathrm{mL}$ ). Aunque no encontramos diferencias estadísticamente significativas para los niveles de adiponectina entre estos dos grupos, es importante notar que hay una menor dispersión de estos valores en el grupo control con respecto al grupo obesidad (Figura 1d). Es necesario llevar a cabo mayor investigación para conocer la razón de esta diferencia en la dispersión pero creemos que esto se encuentra relacionado a los diferentes factores vinculados con el desarrollo de la obesidad, tanto aspectos ambientales como socio-económicos ${ }^{(33)}$, que no se han considerado en esta investigación.

A la fecha, la búsqueda de literatura en PubMed con los términos "adiponectin AND Peruvian" permite encontrar un único estudio con individuos peruanos con respecto a adiponectina y otras citocinas y su relación con periodontitis crónica ${ }^{(38)}$. Aunque el estudio reporta diferencias en los niveles de adiponectina en individuos obesos con respecto a individuos con peso normal, sorprendentemente los niveles altos de adiponectina se encuentran en el grupo de individuos obesos (media $=17,5 \mu \mathrm{g} / \mathrm{mL} ; \mathrm{SD}=11,0 \mu \mathrm{g} / \mathrm{mL}$ ), en comparación con los individuos no obesos (media $=7,2$ $\mu \mathrm{g} / \mathrm{mL} ; \quad \mathrm{SD}=5,8 \mu \mathrm{g} / \mathrm{mL}$ ). Como se ha mencionado anteriormente, creemos que otras variables, no evaluadas en nuestro estudio, así como en el de Mendoza-Azpur et al. ${ }^{(38)}$, tales como el estado socioeconómico, el lugar de residencia (área urbana o rural) o contaminantes ambientales podrían ser importantes factores de confusión que podrían explicar estas diferencias ${ }^{(33)}$.

La fuerza de la correlación inversa entre la concentración de adiponectina y el índice de masa corporal y/o la circunferencia abdominal varía entre las poblaciones estudiadas; por ejemplo, Arita et al. (12) encuentran una correlación "fuerte" entre la concentración de adiponectina y el IMC $(r=-0.66$ en hombres y $r=-0.48$, en mujeres) mientras que Volp et al. ${ }^{(10)}$ reportan una fuerza de correlación mucho menor entre las mismas variables, aunque para hombres y mujeres juntos $(r=-0.178)$. También Okauchi et al. (11) encuentran una correlación inversa con el cambio de adiponectina ( $\Delta$ adiponectina) y el cambio en el IMC $(\triangle I M C)$ por sexo $(r=-0.256$ y $r=-0.223$, en hombres y mujeres, respectivamente). En nuestra investigación, para el grupo control, la única correlación inversa estadísticamente significativa se encontró entre la concentración de adiponectina y la concentración de glucosa $(r=-0.42, P=0.0409)$, no obstante, ya que el IC $95 \%$ del coeficiente del coeficiente de correlación (r) correlación es muy amplio y con un límite superior de -0.02 , sugerimos tomar estos resultados con cautela. 
En este contexto, creemos que es importante subrayar que, aunque los coeficientes de correlación son útiles para describir la fuerza de las relaciones lineales entre variables numéricas, la descripción "estadísticamente significativa" no supone una relevancia clínica de esta relación, de hecho, valores cercanos a cero $(-0.3$ a 0.3$)$ pueden ser considerados como insignificantes ${ }^{(38)}$.

Por otro lado, en el grupo obesidad no encontramos ninguna correlación estadísticamente significativa entre la concentración de adiponectina y el IMC o la circunferencia abdominal. Sin embargo, en este grupo sí encontramos un incremento en los niveles de adiponectina luego del programa (Figura 1d). Independientemente de estas diferencias estadísticamente significativas, es importante notar que el incremento encontrado (1.82) podría ser de baja relevancia considerando que el límite inferior del intervalo de confianza es cercano a cero (0.38). Desafortunadamente, nuestra investigación no ha podido abarcar otros parámetros clínicos que podrían dilucidar la relevancia clínica de esta diferencia. No obstante, la información presentada en esta investigación se suma a los esfuerzos que se realizan para determinar el papel de la adiponectina como biomarcador de cambios metabólicos asociados al desarrollo de obesidad.

En función de nuestros resultados, concluimos que la concentración de adiponectina incrementa cuando la masa corporal se reduce como consecuencia de actividad física aeróbica y dieta baja en calorías en mujeres premenopáusicas.

\section{Agradecimientos}

Agradecemos a todas las personas que decidieron participar en esta investigación. A Patricia Woll Tosso y Julia Sidorova por la revisión y sugerencias en la redacción. Al personal técnico del Servicio de Endocrinología y del Servicio de Terapia Física y Rehabilitación del Hospital Nacional Arzobispo Loayza.

\section{Contribuciones}

WAGQ analizó los datos y redactó el manuscrito.

DTG y CPPR llevaron a cabo todos los experimentos.

FAOLS revisó el proyecto y participó en la solicitud de financiamiento.

JSV y JCT captaron y diagnosticaron a todas las participantes.

MYPA desarrolló el concepto y diseño el proyecto. Lideró el proyecto, analizó los datos y revisó el manuscrito en su versión final.

\section{REFERENCIAS BIBLIOGRÁFICAS}

1. World Health Organization. Obesidad y Sobrepeso [Internet]; 2019. Disponible en: HYPERLINK "http://www.who.int/es/ news-room/fact-sheets/detail/obesity-and-overweight" www. who.int/es/news-room/fact-sheets/detail/obesityand-overweight

2. Fujita K, Hayashi T, Matsushita M, Uemura M, Nonomura N. Obesity, Inflammation, and Prostate Cancer. J Clin Med. 2019 Feb; 8(2):E201.

3. Arroyo-Johnson C, Mincey KD. Obesity Epidemiology Worldwide. Gastroenterol Clin North Am. 2016 Dec; 45(4):571-9.

4. Nimptsch K, Pischon T. Obesity Biomarkers, Metabolism and Risk of Cancer: An Epidemiological Perspective. Recent Results Cancer Res. 2016; 208:199-217.

5. Gandjour A. Cost-effectiveness of preventing weight gain and obesity: what we know and what we need to know. Expert Rev Pharmacoecon Outcomes Res. 2012 Jun; 12(3):297-305.

6. Serafini M, Toti E. Unsustainability of Obesity: Metabolic Food Waste. Front Nutr. 2016 Oct; 3:40.

7. Turer AT, Scherer PE. Adiponectin: mechanistic insights and clinical implications. Diabetologia. 2012 Sep; 55(9):2319-26.

8. Whitehead JP, Richards AA, Hickman IJ, Macdonald GA, Prins JB. Adiponectin-a key adipokine in the metabolic syndrome. Diabetes Obes Metab. 2006 May; 8(3):264-80.

9. Robinson K, Prins J, Venkatesh B. Clinical review: adiponectin biology and its role in inflammation and critical illness. Crit Care. 2011 Apr; 15(2):221.

10. Volp AC, Barbosa KB, Bressan J. Triacylglycerols and body fat mass are possible independent predictors of $\mathrm{C} 3$ in apparently healthy young Brazilian adults. Nutrition. 2012; 28(5):54450.

11. Okauchi Y, Kishida K, Funahashi T, Noguchi M, Ogawa T, Ryo M, et al. Changes in serum adiponectin concentrations correlate with changes in BMI, waist circumference, and estimated visceral fat area in middle-aged general population. Diabetes Care. 2009 Oct; 32(10):e122.

12. Arita Y, Kihara S, Ouchi N, Takahashi M, Maeda K, Miyagawa $J$, et al. Paradoxical decrease of an adipose-specific protein, adiponectin, in obesity. Biochem Biophys Res Commun. 1999 Apr; 257(1):79-83.

13. Katsiki N, Mantzoros C, Mikhailidis DP. Adiponectin, lipids and atherosclerosis. Curr Opin Lipidol. 2017 Aug; 28(4):347-54.

14. Liu D, Li S, Li Z. Adiponectin: A biomarker for chronic hepatitis C?. Cytokine. 2017 Jan; 89:27-33.

15. Shibata R, Ouchi N, Ohashi K, Murohara T. The role of adipokines in cardiovascular disease. J Cardiol. 2017 Oct; 70(4):329-34.

16. Zha D, Wu X, Gao P. Adiponectin and Its Receptors in Diabetic Kidney Disease: Molecular Mechanisms and Clinical Potential. Endocrinology. 2017 Jul; 158(7):2022-34.

17. Divella R, De Luca R, Abbate I, Naglieri E, Daniele A. Obesity and cancer: the role of adipose tissue and adipocytokines-induced chronic inflammation. J Cancer. 2016 Nov; 7(15):2346-59.

18. Wang ZV, Scherer PE. Adiponectin, the past two decades. J Mol Cell Biol. 2016 Apr; 8(2):93-100.

19. Woodward L, Akoumianakis I, Antoniades C. Unravelling the adiponectin paradox: novel roles of adiponectin in the regulation of cardiovascular disease. $\mathrm{Br}$ J Pharmacol. 2017 Nov; 174(22):4007-20.

20. Funahashi T, Nakamura T, Shimomura I, Maeda K, Kuriyama $\mathrm{H}$, Takahashi $M$, et al. Role of adipocytokines on the pathogenesis of atherosclerosis in visceral obesity. Intern Med. 1999 Feb; 38(2):202-6. 
21. Fang CL, Huang LH, Tsai HY, Chang HI. Dermal Lipogenesis Inhibits Adiponectin Production in Human Dermal Fibroblasts while Exogenous Adiponectin Administration Prevents against UVA-Induced Dermal Matrix Degradation in Human Skin. Int J Mol Sci. 2016 Jul; 17(7):E1129.

22. Salman B, Yilmaz TU, Tezcaner T, Demir EO, Pasaoglu OT. Exogenous recombinant adiponectin improves survival in experimental abdominal sepsis. Balkan Med J. 2014 Sep; 31(3):244-8.

23. Abou-Samra M, Boursereau R, Lecompte S, Noel L, Brichard SM. Potential Therapeutic Action of Adiponectin in Duchenne Muscular Dystrophy. Am J Pathol. 2017 Jul; 187(7):1577-85.

24. Ng RC, Chan KH. Potential Neuroprotective Effects of Adiponectin in Alzheimer's Disease. Int J Mol Sci. 2017 Mar; 18(3):E592.

25. Ackerman SE, Blackburn OA, Marchildon F, Cohen P. Insights into the Link Between Obesity and Cancer. Curr Obes Rep. 2017 Jun; 6(2):195-203.

26. Francisco C, Neves JS, Falcao-Pires I, Leite-Moreira A. Can Adiponectin Help us to Target Diastolic Dysfunction? Cardiovasc Drugs Ther. 2016 Dec; 30(6):635-44.

27. R Core Team. A language and environment for statistical computing [Internet]; 2018. Disponible en: https://www.rproject.org/

28. R Studio Team. Integrated Development for R [Internet]; 2015. Disponible en: http://www.rstudio.com/

29. Abranches MV, Oliveira FCE, Conceicao LL, Peluzio MD. Obesity and diabetes: the link between adipose tissue dysfunction and glucose homeostasis. Nutr Res Rev. 2015 Dec; 28(2):121-32.

30. Ghantous CM, Azrak Z, Hanache S, Abou-Kheir W, Zeidan A. Differential Role of Leptin and Adiponectin in Cardiovascular System. Int J Endocrinol. 2015; 2015:534320.

31. Hui X, Lam KS, Vanhoutte PM, Xu A. Adiponectin and cardiovascular health: an update. Br J Pharmacol. 2012 Feb; 165(3):574-90.

32. Kontny E, Janicka I, Skalska U, Maslinski W. The effect of multimeric adiponectin isoforms and leptin on the function of rheumatoid fibroblast-like synoviocytes. Scand J Rheumatol. 2015; 44(5):363-8.

33. Ford ND, Patel SA, Narayan KM. Obesity in Low-and MiddleIncome Countries: Burden, Drivers, and Emerging Challenges. Annu Rev Public Health. 2017 Mar; 38:145-64.

34. Goto A, Noda M, Goto M, Yasuda K, Mizoue T, Yamaji T, et al. Plasma adiponectin levels, ADIPOQ variants, and incidence of type 2 diabetes: A nested case-control study. Diabetes Res Clin Pract. 2017 May; 127:254-64.

35. Ntzouvani A, Fragopoulou E, Panagiotakos D, Pitsavos C, Antonopoulou S. Reduced circulating adiponectin levels

are associated with the metabolic syndrome independently of obesity, lipid indices and serum insulin levels: a crosssectional study. Lipids Health Dis. 2016; 15(1):140.

36. Lee S, Kwak HB. Role of adiponectin in metabolic and cardiovascular disease. J Exerc Rehabil. 2014 Apr; 10(2):54-9.

37. Hebbard L, Ranscht B. Multifaceted roles of adiponectin in cancer. Best Pract Res Clin Endocrinol Metab. 2014 Jan; 28(1):59-69.

38. Mendoza-Azpur G, Castro C, Pena L, Guerrero ME, De La Rosa $M$, Mendes $C$, et al. Adiponectin, leptin and TNF- a serum levels in obese and normal weight Peruvian adults with and without chronic periodontitis. J Clin Exp Dent. 2015 Jul; 7(3):e380-6.

39. Mukaka MM. Statistics corner: A guide to appropriate use of correlation coefficient in medical research. Malawi Med J. 2012 Sep; 24(3):69-71.

Fuentes de financiamiento

Este trabajo fue financiado por el Proyecto Contrato $\mathrm{N}^{\circ}$ 125-2010-CONCYTEC-OAJ y la Universidad de San Martín de Porres (USMP).

Conflictos de interés

Los autores declaran no tener ningún conflicto de interés.

\section{Correspondencia:}

Mónica Yolanda Paredes Anaya

Dirección: Laboratorio de Genética de Enfermedades Metabólicas $\left(N^{\circ} 115\right)$, Facultad de Ciencias Biológicas, Universidad Nacional Mayor de San Marcos. Ciudad Universitaria, Lima 1, Perú.

Teléfono: +51 6197000

Correo electrónico: mparedesa1@unmsm.edu.pe

$$
\begin{array}{llllll}
\text { Recibido: } & 02 & \text { de } & \text { abril de } & 2019 \\
\text { Evaluado: } 08 \text { de } & \text { abril de } & 2019 \\
\text { Aprobado: } & 31 & \text { de mayo de } & 2019
\end{array}
$$

(c) La revista. Publicado por Universidad de San Martín de Porres, Perú. (сc) ву Licencia de Creative Commons Artículo en acceso abierto bajo términos de Licencia Creative Commons Atribución 4.0 Internacional. (http: //creativecommons.org/licenses/by/4.0/)

\section{ORCID iDs}

Wilser Andrés García-Quispes Dina Torres Gonzales Carlos Patricio Padilla Rojas Frank Lizaraso-Soto Mónica Yolanda Paredes Anaya https://orcid.org/0000-0001-6628-2985 (D) https: / / orcid.org/0000-0003-2142-7575 (1) https: / / orcid.org/0000-0002-0562-0431 (D) https: / / orcid.org/0000-0001-9993-9998 (1D) https: / / orcid.org/0000-0002-9038-2270 